\title{
Modelling and Design of Photonic Bandgap Devices: a Microwave Accelerating Cavity for Cancer Hadrontherapy
}

\author{
Roberto Marani and Anna Gina Perri \\ Politecnico di Bari, Dipartimento di Elettrotecnica ed Elettronica \\ Bari, Italy
}

\section{Introduction}

In recent years hadrontherapy became one of the most important way to fight against deepsided cancer. This technique had been theoretically studied and now it seems to be the main method able to replace radiosurgery, which is not always incisive and sometimes leads up to side effects. These results can be obtained by exploiting the capability of accelerated hadrons (heavy particles) to hit ill tissue with high accuracy, without any damage to neighbour tissues. Nevertheless the high dimensions and costs of the present structures, implemented to accelerate hadrons, forbid the actual use of hadrontherapy in oncology. The research aim is concerned with the definition of new techniques able to develop integrated systems that can accelerate and control particle beams to improve performance and that can decrease the cost of classical medical accelerators.

By analysing the energy transmission profile of accelerated protons and ions through water, which is the main component of a biological tissue of the human body, it is possible to understand that hadrons can easily reach a penetration depth between 25 and $30 \mathrm{~cm}$, discharging the maximum value of energy in a small volume at the travel ending. This maximum is also called Bragg peak. The protons beam energy can be modulated in order to get the exact depth where the cancer tissue is localized. If the cancer thickness is higher than the Bragg peak extension, a SOBP (Spread-Out Bragg Peak) could be realized by adding an appropriate number of power peaks of a decreasing intensity. In this case it is possible to create a peak of the desired depth, equal to that of the cancer mass.

This fundamental quality, together with the low lateral diffusion that happens when a hadron beam flows inside a biological tissue, allows a millimeter accuracy and consequently the possibility to realize an actual radiotherapy with a deep continuous scanning by varying the particles energy and the beam localization along the lateral directions, by a translation of the incident beam.

The high selectivity of these treatments, comparable to a surgical intervention (radiosurgery), allows to attack cancer cells without damages of near healthy tissues. Moreover this characteristic is fundamental if the cancer mass is localized near a vital organ or in a critical area. 
In this chapter, after a review about the principal characteristics and applications of PBG devices, we carefully describe the design of a microwave PBG-based accelerating cavity for cancer hadrontherapy. The prototype has been fabricated and experimentally characterized obtaining confirmation of expected theoretical results.

\section{PBG-based accelerating cavity for medical applications}

One of the most important issue in hadrontherapy is related to the choice of the particle accelerator, that are typically classified in cyclotrons and synchrotrons. A hospital accelerator should obey some specifications different from the conventional rules of a research system. All beam parameters, such as energy variation and current intensity, have to be optimized for clinical applications. The main specification for a correct design of the accelerating system are reliability, flexibility and, finally, low fabrication and maintenance costs. The control system plays a fundamental role in meeting the quality requirements: it has to be user-friendly and full integrated in the security system of the whole apparatus.

Typical energy values for protons beam are estimated to be in a range between 60 and 250 $\mathrm{MeV}$ (Coutrakon et al., 1999), which implies a path inside the biological tissue that spans from $3.1 \mathrm{~g} / \mathrm{cm}^{2}$ to $38.3 \mathrm{~g} / \mathrm{cm}^{2}$. It is thus necessary to get high acceleration gradient and energy values whatever particle accelerator is used.

Traditional particle accelerators can be considered as metallic waveguides which carry $\mathrm{TM}_{01}$ mode, thus producing the highest acceleration for a given working power, but suffering from the excitation of higher order modes (HOM) at high frequencies. Moreover, metallic walls produce absorption losses that increase with the frequency (Shapiro et al., 2001).

The PBG-based resonant cavities are used in particle accelerator applications, with a drastic improvement of performances. In fact:

- PBG materials can substitute the metallic walls to achieve perfectly reflective surfaces, without absorption losses;

- $\quad$ PBG structures allow the suppression of higher order modes in the resonant cavity of the accelerator;

- Since the field in a PBG-based cavity is strongly confined in a very small region, wider tolerances become acceptable for the material quality, which has to be very high in the centre of the cavity - where the field is confined - but can be lower in the external region. This aspect is very important when a superconductor material is used, since semiconductors have not a uniform high quality on large surfaces;

- Straight structures can be realized and high accelerations obtained;

- It is possible to optimize the coupling between the resonant cavity and the input waveguide, thus reducing the resonance frequency shift which is a typical problem of a standard pillbox cavities.

Moreover the presence of a defective region, in which the periodicity is not regular because one or more rods are removed, produces a strong electromagnetic field localization at a given frequency, which depends on the characteristics of the defect. The presence of the defect allows resonant effects with high value of quality factor and high suppression of higher order modes.

The main design parameters are: height, diameter and rods number, distance among the rods centres, geometry and thickness of plates. Anyway, the design statements are related to the application of these accelerant cavities. 
To explain carefully the design procedure of the proposed microwave PBG-based accelerating cavity for cancer hadrontherapy, it is necessary a brief review about the principal characteristics of PBG crystals and their applications to the PBG devices design.

\section{Principal Characteristics of Photonic Crystals}

Photonic crystals are materials able to influence the light propagation analogously it occurs for the propagation of the electrons in semiconductors (Perri, 2007).

In fact, in a semiconductor the crystal lattice causes the formation of a periodic potential for an electron which propagates through it. This periodicity determines the formation of energy bandgaps for the electronic states, where the propagation of electrons is forbidden.

In photonic crystals a principle similar to what seen for the semiconductor crystals is exploited for the propagation of some light quanta (or photons). In this case the crystal periodicity is artificially realized by means of an alternation of dielectric macroscopic materials.

According to their geometrical characteristics, photonic crystals inhibit the light propagation in one or more directions, depending on the working frequency. This leads to the existence of a bandgap, i.e. a frequency range in which the wave can not propagate. The bandgap is complete if the propagation is prevented in all possible directions at those frequencies.

Fig.1 shows different PBG based structures, characterized by one-dimensional (1D), twodimensional (2D) or three-dimensional (3D) periodicity, each of them infinitely extended and then so-called "bulk". On the contrary, Fig. 2 shows waveguiding periodic structures, characterized by a finished thickness and a 1D, 2D or 3D periodicity.
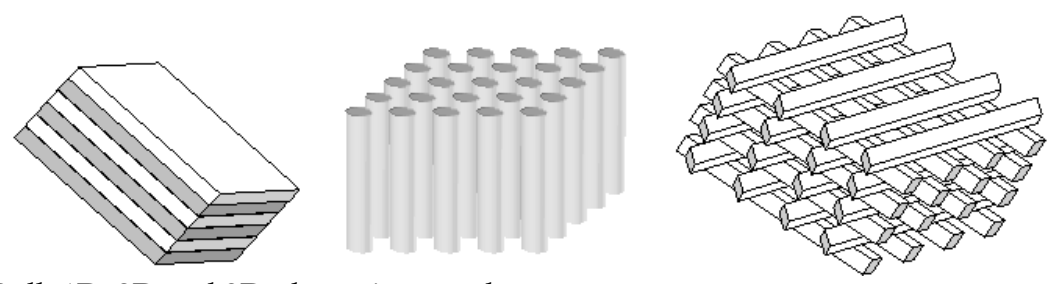

Fig. 1. Bulk 1D, 2D and 3D photonic crystals.
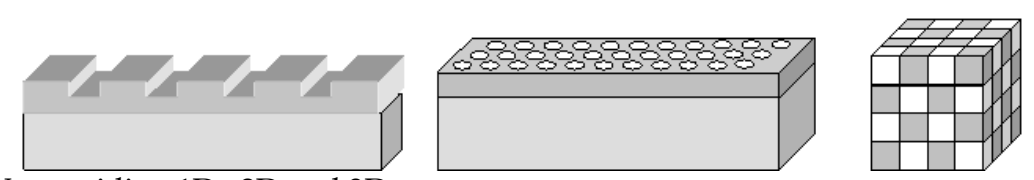

Fig. 2. Waveguiding 1D, 2D and 3D structures.

A wave that impinges on a dielectric discontinuity is partially reflected, partially transmitted and partially diffracted. We can then distinguish between two waves, one propagating in a positive direction and the other in a negative direction. If the wave is incident on a periodic structure, the produced waves can - in particular conditions destructively interfere and propagation is then inhibited, so a bandgap appears. 
The characterization of a photonic crystal is carried out through the so called Brillouin diagrams, where the frequency of the allowed electromagnetic modes is plotted as a function of the normalized propagation constant in all directions of the irreducible Brillouin zone.

Fig. 3 shows a frequency range (i.e. bandgap), between the dashed lines, in which no propagation can occur.

The frequency range in which the wave is particularly concentrated in the high refractive index medium is called dielectric band, while the other one is called air band.

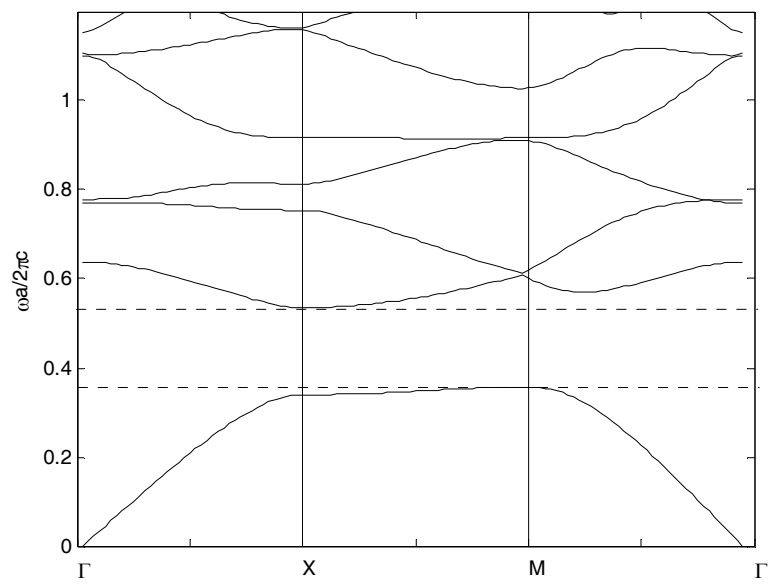

Fig. 3. Photonic band of a PBG structure having lattice constant $a$ and with different thickness layers. The thickness of the layers at high index is $0.2 a$ and that of the layers at low index is $0.8 a$.

Moreover, a lattice of circular rods is shown in Fig.4.
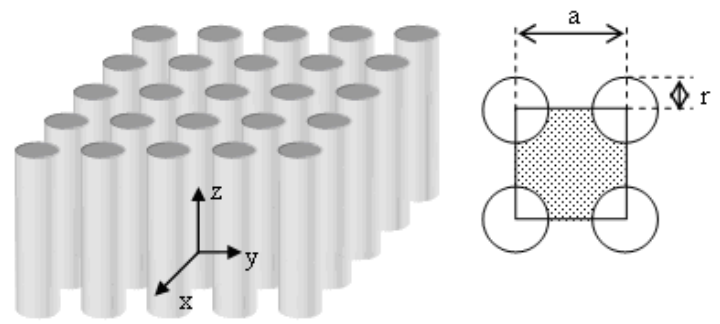

Fig. 4. 2D PBG structure.

The material is homogeneous in the $z$ direction and periodic in the $x y$-plane, being $a$ the lattice constant. The grating is arranged accordingly with a square cell array, as shown in the inset of the same Fig. 4.

The physical characteristics of the described structure are determined by its design parameters: the lattice constant, the filling factor or ratio, the rod radius, the geometry of the unit cell, the refractive index contrast. 
The filling factor gives a measure of the electromagnetic energy located inside the high index dielectric regions in comparison with the energy distributed in the whole volume, being large in the dielectric band and small in the air band.

The photonic investigation of the described structure, having rod radius equal to $0.2 a$, shows a different behaviour for TE and TM modes, as depicted in Fig. 5. In particular, there is a complete bandgap for TM modes, while TE modes can always propagate for each frequency.

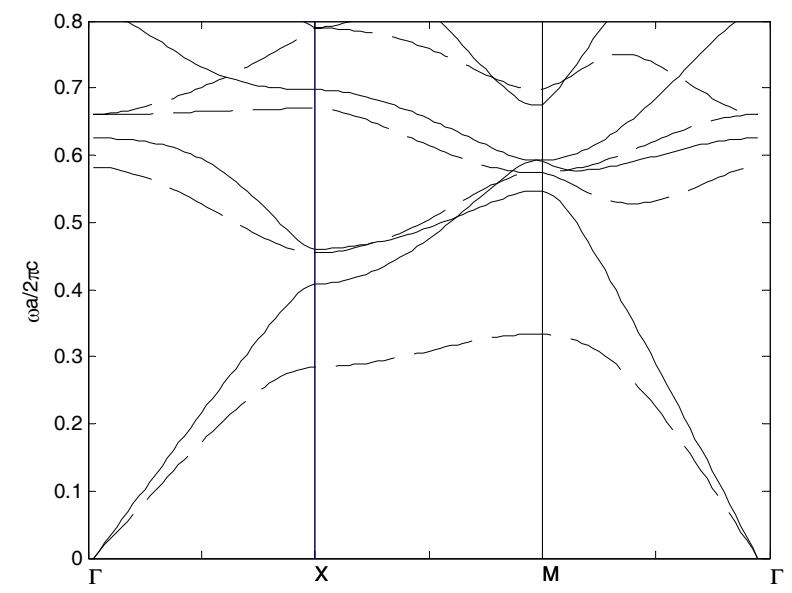

Fig. 5. Brillouin diagram for a squared array of dielectric columns having $r=0.2 a$ for: TE modes (solid line) and TM modes (dashed line).

The structure, composed by a square array of high dielectric columns, has a complete bandgap for the TM modes but not for the TE ones for the following reasons:

- the mode mainly localized in the high refractive index medium has a frequency lower than that of the mode concentrated in the low refractive index medium: this explains the bandgap formation (separation in $\omega$ );

- $\quad$ TM modes have the Maxwell displacement vector $D$ orthogonal to the periodicity plane and, then, localized in the high refractive index regions;

- for the TE modes the Maxwell displacement vector $D$ is oriented in the periodicity plane and must necessarily enter into the low index regions (for both the air and dielectric bands), thus resulting in a smaller separation among the bands in comparison with the TM ones.

The wave behaviour changes if the type of used structures varies.

For example a structure with square section rods at low index has characteristics, in terms of bandgap, opposite to those of a structure with circular section rods. In fact:

- the squared structure offers a continuous path among the high index regions: the field, orthogonal to the periodicity plane in the TM modes, is concentrated in the crossings among the high index bars (dielectric band) or inside the high index bars (air band). It follows that the field configuration is similar for both the bands and, so, the separation between them is small; 
- $\quad$ for the TE modes the $D$ field is contained in the periodicity plane and localizes inside the high index regions in the dielectric band, while, presenting nodes in the high index bars, it must necessarily penetrate into the low index regions to place itself in the air band structure, with a consequent greater bandgap in comparison to the TM case.

According to the investigated structures, design rules, useful to establish the necessary conditions for a complete bandgap, can be determined:

- not connected regions of high refractive index materials improve the bandgap for TM modes;

- connected regions of high refractive index material improve the bandgap for TE modes;

- an hexagonal cell of air columns carries out both the previous characteristics, thus providing a complete bandgap for both polarizations;

- for strong index contrasts even a square cell of air columns presents a complete bandgap.

The following photonic band diagram (Fig. 6), calculated for a structure having a triangular cell of air columns drilled in a semiconductor substrate, shows a complete bandgap for both TE and TM modes.

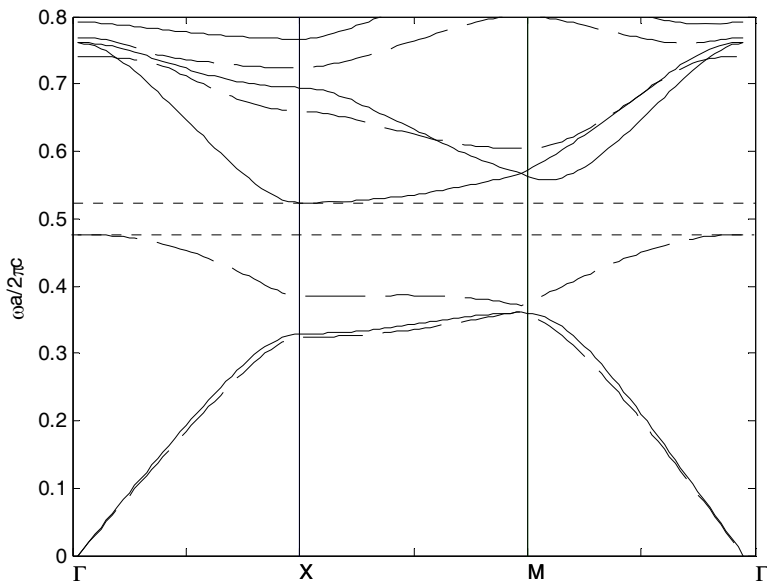

Fig. 6. Photonic diagram for a structure having a triangular cell of air columns drilled in a semiconducor substrate. A bandgap for both polarizations is shown.

A 3D crystal has an almost infinite numbers of geometries, but only few of them having a complete bandgap. A typical 3D structure is the opal (Fig. 7), constituted by high index spheres in a low index background or vice versa (inverted opal). The spheres can be arranged in a suitable manner, thus reproducing a general crystal structure. 
Fig. 7. 3D PBG opal.

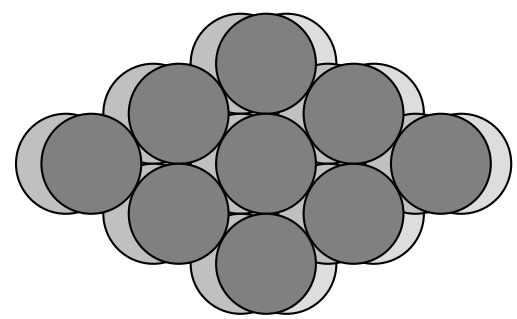

\section{Defects and devices on PBG}

The introduction of defects in a photonic crystal determines the forming of photonic states located in the gap. Such characteristic is exploited to carry out devices with high capabilities, for example optical microresonators (where a column is removed) or low losses waveguides, based on the presence of a bandgap instead of the total internal reflection.

The behaviour of a defective photonic crystal is analogous to that of a doped semiconductor, which is characterized by the presence of allowed states for the electrons inside the forbidden band near the conduction or the valence bands, depending on the kind of impurities embedded in the semiconductor.

The presence of the defects in 1D, 2D and 3D structures is analyzed in following subsections.

\section{4.a Defects in 1D structures}

The defect is constituted by a missing layer or a layer having a width different from the others. This situation is depicted in Fig.8.
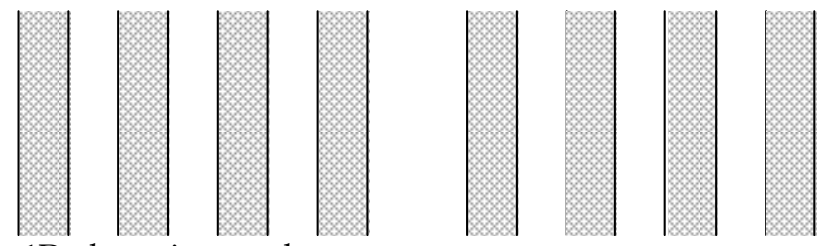

Fig. 8. Defect in a 1D photonic crystal.

If the defect has a suitable width, an allowed state is provided within the bandgap, thus resulting in a strong localization of the mode, which exponentially vanishes into the surrounding periodical regions.

The presence the 1D defect provides a phase shift of an odd multiple of $\pi$ for a complete oscillation (backwards and forwards).

It follows that the allowed states exist only at discrete energies and the corresponding resonance frequencies decrease with an increasing defect size.

The transmission curve is also modified at the allowed state wavelength $(\lambda)$ showing a maximum, as depicted in Fig. 9. 


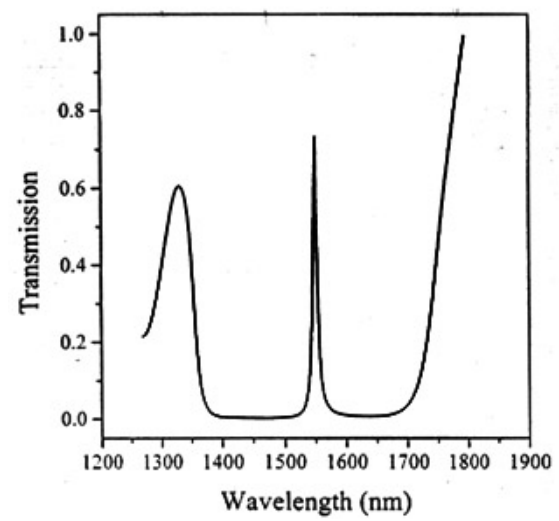

Fig. 9. Transmission coefficient in presence of a defect to $\lambda / 4$.

Fabry-Perot passband filters can be also designed by using defective 1D PBG based structures.

\section{4.b Defects in 2D structures}

In two-dimensional photonic crystals a single defect is achieved by removing a rod from the lattice (Fig. 10) or replacing it with another one of different refractive index material (Fig. 11).

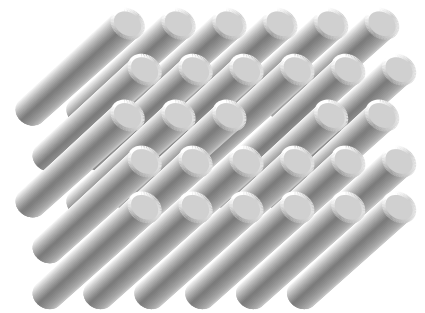

(a)

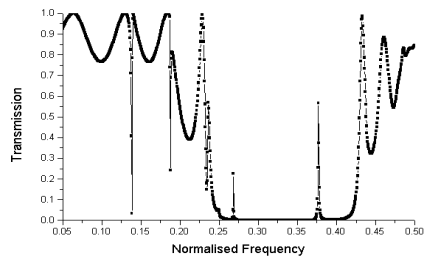

(b)

Fig. 10. (a) 2D photonic crystal with defect obtained by removing a dielectric rod; (b) Transmission spectrum.

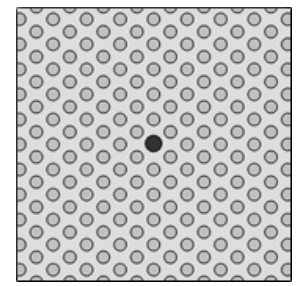

Fig. 11. 2D defect obtained by using an high refractive index rod. 
The presence of a defect determines a transmission peak inside the forbidden band and produces a concentration of field in the defective region, thus creating a two-dimensional cavity surrounded by reflecting walls supporting an allowed state inside the bandgap.

In a 2D PBG, unlike a one-dimensional crystal, line defects can be realized by removing one or more sets of rods.

A waveguide is realized by making a defective path, where the light can be confined since no propagation in the surrounding crystal can occur, thus decreasing propagation losses. This feature can be advantageously used to realize strongly curved waveguides.

Fig. 12 shows the architecture of several PBG-based optical devices.

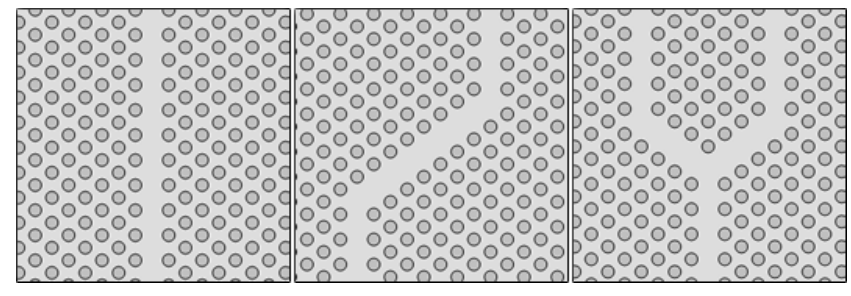

(a)

(b)

(c)

Fig. 12. Different structures realized with line defects: (a) straight; (b) curved waveguide; (c) Y-branch.

\section{4.c Defects in 3D structures}

It is possible to introduce some irregularities in the grating of a 3D crystal:

- by adding a material with different dielectric constant in the unitary cell (dielectric defect);

- by removing part of the dielectric material from the unitary cell air defect.

In the first case the defect behaves analogously to a donor atom in a semiconductor crystal, thus producing an allowed state (donor mode) near the lower edge of the air band.

Likewise, an air defect is similar to an acceptor atom, and localizes the field distribution (acceptor mode) near the upper edge of the dielectric band. These defects are particularly interesting for realizing resonant microcavities with high quality factor $\mathrm{Q}$.

Some theoretical investigations (Yablonovich, 1994) show that the frequency of the acceptor mode increases with an increasing volume of removed material, while a small amount of volume results in an acceptor level near the edge of the dielectric band. Moreover, a too small amount of removed material pushes localized states within a continuum of levels below the top of the dielectric band.

Similarly, a donor level requires a minimum removed volume for creating an allowed level below the air band edge. In this case, the frequency of the donor level diminishes with increasing the defect volume.

In a 3D crystal it is possible to localize the light in a single region introducing a point defect. This behaves as an entirely reflecting walls resonant cavity, where the electromagnetic field is confined. Outwardly the defect, the field will vanish along the three crystal dimensions.

Besides point defects, linear type defects can also be realized by creating a defective region in the crystal. This approach allows to fabricate high quality waveguides, able to confine the light and to make negligible losses also at optical frequencies. 


\section{Applications of Photonic Bandgap Crystals}

The first PBG crystal was fabricated by Yablonovitch in 1989 at Bell Communications Research in New Jersey, characterized by a bandgap only at microwave frequencies because of technological limitations (to work with smaller wavelengths a smaller rod size is required). The $2 \mathrm{D}$ bulk crystal was obtained by drilling the silicon according a face centred cubic (FCC) arrangement, being this architecture the simplest and most suitable for achieving a complete bandgap (in all directions and for all polarizations), as suggested by theoretical studies.

Currently, 2D structures are most investigated at optical frequencies to realize waveguides and monolithic integrated optical circuits.

The most important applications are:

- $\quad$ waveguides, power splitters and switches with low losses over long distances and in presence of strong bends;

- optical fibres, monomodal in a wide range of wavelength, with a low core refractive index. Only the mode that satisfies the Bragg condition can propagate;

- $\quad$ perfectly reflective mirrors, in particular for laser cavity walls;

- LED diodes having very high external efficiency (4\% without PBG) because only the emission of the transmittable modes occurs. All the emitted energy is then transmitted;

- laser diodes having low threshold $(<100 \mu A)$ : since the spontaneous emission is suppressed, because photons having energy inside the band-gap are not emitted, the related loss decreases, the efficiency increases, the dissipated power decreases;

- narrow band filters for DWDM (Dense Wavelength Division Multiplexing) systems;

- $\quad$ resonant cavities with very high $Q$-factor;

- biomedical sensing applications based on porous silicon;

- $\quad$ particle physics applications to realize high spectral purity accelerators;

- $\quad$ photonic integrated circuits: PBG allow to reduce photonic circuits sizes (up to a few hundred of squared $\mu \mathrm{m}$ ) because:

- laser beam can propagate through strongly bent guides with very low losses;

- LED and laser high efficiency allows a low power consumption and, then, the integration in very small areas;

- the power coupling among adjacent waveguides is strongly reduced;

- superprism effects can be used.

In particular, in the following sub-sections, we examine the PBG applications related to resonant cavities and particle accelerators.

\section{5.a Resonant cavities}

Conventional microwave resonant cavities are boxes enclosed by conductive walls containing oscillating electromagnetic fields. Conductive walls act as perfect screens, therefore avoiding any radiation of energy away from the box. Because of the large extension of inner walls, current density and losses are reduced.

Optical resonators are rather different from the microwave typical ones for the smaller operative wavelength, which yields a large number of allowed modes. Although there are 
several and important differences between optical and microwave resonators, some parameters, as quality factor $Q$, are very useful for both kind of cavities. The quality factor $Q$ is defined as:

$$
\mathrm{Q}=\omega_{0} \mathrm{U} / \mathrm{W}
$$

where $\omega_{0}$ is the resonance frequency, $U$ the electromagnetic energy stored in the cavity and $W$ the lost power. Losses in the dielectric material and radiations from small apertures can cause the lowering of $Q$. The $Q$ factor allows to evaluate also the filter bandwidth, defined as:

$$
\Delta \omega / \omega_{0} \cong 1 / Q
$$

where $\Delta \omega$ is the range between two frequencies at which the signal power is $3 d B$ lower than the maximum value. It can be shown that, at a given frequency, the $Q$-factor increases with an increasing order of mode.

As seen in the previous paragraph, a resonant cavity can be obtained by introducing a defect in a photonic crystal in order to modify its physical properties. In the case of a defectless structure, electromagnetic waves can not propagate when the operative frequency is inside the bandgap, in which a narrow band of allowed frequencies can be achieved breaking the crystal periodicity through a suitable defect.

Light localization is used in the PBG based microcavity design to optimize the $Q$-factor, which depends on the geometrical and physical properties of the defect. Lattice defects are constituted by dielectric regions of different shapes, sizes or refractive index values. By changing one of these parameters in the defective region we can modify the mode number of the resonance frequency inside the cavity. Moreover, the spectral width of the defect mode is demonstrated to decrease rapidly with an increasing number of repetitions of the periodic structure around the cavity region, so improving the selectivity of the resonance frequency inside the bandgap.

The excellent performances of PBG structures have been used to develop resonators characterized by high values of $Q$-factor working at microwave frequencies, by introducing defects in 3D and particularly in 2D structures. Microwave resonant cavities are constituted by dielectric materials and metals, thus keeping the same fundamental properties of the PBG structures. Metallic structures are easier and less expensive to realize and can be used for accelerator-based applications. Most interesting 2D and 3D devices have a geometrical structure that allows a large bandgap, achieved by using a triangular cell for 2D or woodpile cell for 3D structures, with an efficient wake-field suppression at higher frequencies, without interfering with the working mode.

In microwave applications the use of carbon based low losses materials (Duroid, Teflon), aluminium oxide or highly resistive silicon is already described in literature. In particular highly resistive silicon has been demonstrated to be most suitable at frequencies near 100 $\mathrm{GHz}$ (Kiriakidis \& Katsarakis, 2000). Moreover, both dielectric and metallic-dielectric gratings have been investigated, thus achieving an improvement in terms of $Q$-factor.

The final architecture is constituted by a 2D triangular lattice, in which a rod at the centre has been removed (defect), thus producing a resonant cavity (Fig. 13). To localize the mode, 
three rows of rods have been used, and all the rods are confined inside a metallic cylinder closed on both ends.

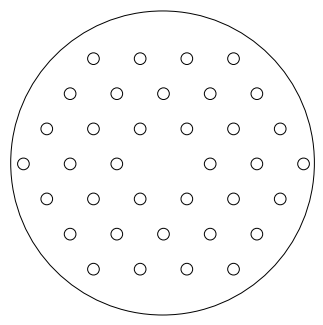

Fig. 13. Architecture of the PBG cavity.

The main difference with traditional cavities is the absence of coupling holes, at the opening of waveguide, which produce a down-shift frequency of $2 \%$. PBG-based cavities are not affected by this problem because of the distributed cavity coupling. In fact, fields are confined by the rods nearest to the defect, and these rods are not perturbed in order to obtain the coupling.

The main steps required to design a resonant cavity are:

- design of the periodic structure to obtain a suitable bandgap around the required working frequency;

- creation of a defect in the grating to establish a defect mode;

- analysis of higher order modes that have to be not confined, being in the crystal passband, and thus can be absorbed by coatings at the edge of the structure;

- design of a suitable hole in the central region of the plates to allow the propagation of the accelerated beam outside the device.

\section{5.b Particle accelerators}

Traditional particle accelerators can be considered as metallic waveguides that carry $\mathrm{TM}_{01}$ mode, thus producing the highest acceleration for a given working power, but even suffering from the excitation of higher order modes (HOM) at high frequencies. Moreover, metallic walls produce absorption losses that increase with the frequency (Shapiro et al., 2001).

PBG-based resonant cavities are used in particle accelerator applications, with drastic improvement of performances. (Fig. 14).

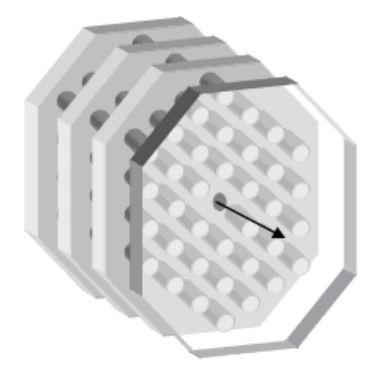

Fig. 14. PBG based particle accelerator architecture. 
The structure is formed by three triangular cell gratings, separated by superconductor layers. Each grating has a defect, obtained by removing a rod. The hole at the centre of conductor layers allows the particle beam emission.

In fact:

- PBG can substitute the metallic walls, obtaining perfectly reflective surfaces without absorption losses;

- PBG allows the suppression of higher order modes in the resonant cavity of the accelerator;

- $\quad$ since the field in a PBG based cavity is strongly confined in a very small region, wider tolerances become acceptable on the material quality, which has to be very high in the centre of the cavity - where the field is confined - but can be lower in the outer regions. This aspect is very important when a superconductor material is used, since semiconductors have not a uniform high quality on large surfaces;

- $\quad$ straight structures can be realized and high accelerations obtained;

- $\quad$ it is possible to optimize the coupling between the resonant cavity and the input waveguide, thus reducing the resonance frequency shift which is a typical problem of a standard pillbox cavities.

The presence of a defective region, in which the periodicity is not regular because one or more rods are missing, produces a strong electromagnetic field localization at a given frequency, which depends on the characteristics of the defect. The bandwidth of the defect is related to the $Q$-factor, so it is possible to make resonators with high $Q$-value and high suppression of the higher order modes.

The main design parameters are: height, diameter and rods number, distance between rods centres, geometry and thickness of plates. In any case, the design statements are related to the application of these accelerant cavities.

\section{Design of PBG-based Accelerating Cavity}

In order to take into account all effects due to the shape of the accelerating cavity, we consider two different architectures both constituted by either dielectric or metallic rods arranged according a 2D periodic triangular lattice, embedded in air and sandwiched between two ideal metal layers. In this way only TM modes are excited. The investigated structures are shown in Fig. 15.

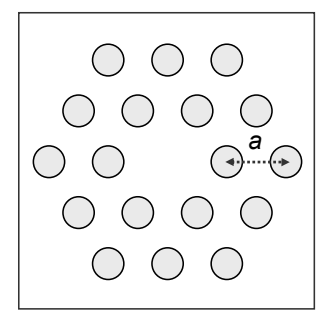

(a)

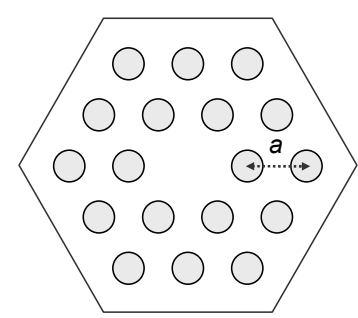

(b)

Fig 15. Accelerator with squared external wall (a) and hexagonal external wall (b).

The aim of the analysis is to find the optimal geometrical parameters for placing the operating resonance frequency close to the centre of the bandgap. Once the lattice 
parameters have been determined, the central rod must be removed to create the resonance condition, thus providing a localized state inside the bandgap.

The analysis makes use of a rigorous formulation of the Quality Factor according to the Floquet-Bloch formalism, to investigate the photonic behaviour of the resonant cavity.

We assume rod radius $R$, lattice constant $a$ (see Fig. 15) and rod height $t_{\text {g. }}$.

To evaluate the Q-factor, defined according to Eqn. (1), it is necessary to calculate the energy $U$ stored by the electromagnetic field and the lost power $W$.

The electromagnetic field energy is given by:

$$
\mathrm{U}=\frac{\mu_{0}}{2} \int_{\mathrm{V}} \mathrm{H}^{2} \mathrm{dv}
$$

where $H$ is the magnetic field amplitude, $\mu_{0}$ is the vacuum permittivity and the integral is extended over the cavity volume. Since the periodic structure is sandwiched between two ideal metal layers, only TM modes can be excited, being the electric field perpendicular to the periodicity plane and all the field components constant with respect to the cavity height. In this case the relationship (3) can be rearranged as:

$$
\mathrm{U}=1 \frac{\mu_{0}}{2} \int_{\mathrm{S}} \mathrm{H}^{2} \mathrm{ds}
$$

where $l$ is the height of the cavity and $S$ is the cavity cross section.

The lost power $W$ can be written as follows:

$$
\mathrm{W}=\mathrm{l} \frac{1}{2} \mathrm{R}_{\mathrm{s}} \int_{\mathrm{l}_{\mathrm{i}}} \mathrm{H}^{2} \mathrm{dl}+2 \frac{1}{2} \mathrm{R}_{\mathrm{s}} \int_{\mathrm{S}_{\wedge}} \mathrm{H}^{2} \mathrm{ds}
$$

where $R_{s}$ is the metal surface resistance. In the previous relationship the first term takes into account the lost power due to the currents on the rods, while the second term evaluates the losses due to currents on the metal layers. By putting:

$$
\begin{gathered}
\delta=\frac{2 \mathrm{R}_{\mathrm{s}}}{\omega \mu_{0}} \\
\mathrm{R}_{\mathrm{eff}}=2 \frac{\int_{\mathrm{S}_{\wedge}} \mathrm{H}^{2} \mathrm{ds}}{\int_{\mathrm{l}_{\mathrm{i}}} \mathrm{H}^{2} \mathrm{dl}}
\end{gathered}
$$

we obtain:

$$
\mathrm{Q}=\frac{1}{\delta\left(\frac{1}{\mathrm{R}_{\mathrm{eff}}}+\frac{1}{1}\right)}
$$


This relationship can be used also for superior order modes supported by the structure, if all fields are constant along the rod height.

The $Q$ factor can be written as:

$$
\frac{1}{\mathrm{Q}}=\frac{1}{\mathrm{Q}_{\delta}}+\frac{1}{\mathrm{Q}_{\mathrm{met}}}
$$

where $Q_{\delta}$ is the quality factor taking into account losses in the dielectric medium, while $Q_{\text {met }}$ accounts for the ohmic losses due to the currents on metallic walls. Moreover:

$$
\mathrm{Q}_{\delta}=\frac{1}{\mathrm{k} \times \tan \delta}
$$

where $k$ is the fraction of the energy stored in dielectric rods, while $\tan \delta$ is the loss tangent due to the dielectric medium. For our calculations we have assumed $\tan \delta$ is equal to $10^{-4}$. $Q_{\text {met }}$ can be calculated by using Eqn. (8), where $R_{\text {eff }}$ is defined in a different way, since the integral at the denominator of Eqn. (7) has to be extended only on the external edge.

\section{Numerical results}

As first step we have investigated the physical properties of a microwave 2D periodic structure in terms of forbidden frequencies.

The designed parameters values are: $a=8.58 \mathrm{~mm}, R=1.5 \mathrm{~mm}, t_{g}=4.6 \mathrm{~mm}, \varepsilon_{a}=9, \varepsilon_{b}=1$. The photonic band diagram shows the first bandgap extending from $12.7 \mathrm{GHz}$ to $20.15 \mathrm{GHz}$. In order to take into account the defect presence, constituted by a rod missing (see Fig. 15), several simulation have been performed by using a FEM (Finite Element Method) based approach (Dwoyer et al., 1988), thus computing both field distributions and $Q$-factors for different configurations.

Fig. 16 shows the first three modes for the electric field $E_{z}$ component.

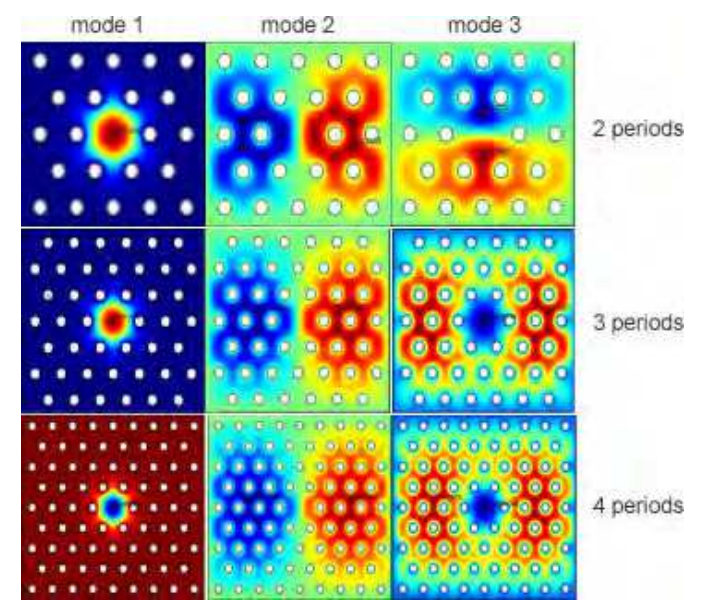

Fig. 16. Electric field component $E_{z}$ for 2,3 and 4 grating periods. 
Modes have been computed for two, three and four grating periods, not showing any difference in the first mode which is well confined in the defect space also for two grating periods. Of course the increase of grating periods does not change the distribution of the first mode, but becomes very significant for high order modes which are distributed externally with respect to the defect space and suffer from losses due to the third grating period.

This aspect can also be noticed from Table 1, in which two different accelerators are compared, the first one with external squared wall (Fig. 15a), the second one with external hexagonal wall (Fig. 15b). Both accelerators have the same periodic structure with metallic rods. In the first column of the Table I the number of grating periods is reported. The change of both the first mode resonant frequency and quality factor with increasing the period number is negligible. On the contrary, high order modes are external to the defect and suffer from any further grating period thus producing an additional loss and a consequent decrease in the $Q$-factor.

\begin{tabular}{|l|l|l|l|l|l|}
\hline & & \multicolumn{2}{|l|}{ Squared wall } & \multicolumn{2}{l|}{ Hexagonal wall } \\
\hline $\mathrm{N}$ & mode & $\begin{array}{l}\text { Frequency } \\
(\mathrm{GHz})\end{array}$ & $\mathrm{Q}$ & $\begin{array}{l}\text { Frequency } \\
(\mathrm{GHz})\end{array}$ & $\mathrm{Q}$ \\
\hline \multirow{3}{*}{2} & 1 & 14.1594 & 4434.3 & 14.1598 & 4439.0 \\
\cline { 2 - 6 } & 2 & 20.5393 & 3721.5 & 21.0130 & 4091.0 \\
\cline { 2 - 6 } & 3 & 20.8323 & 3893.7 & 21.0130 & 4091.7 \\
\hline \multirow{4}{*}{4} & 1 & 14.1592 & 4436.2 & 14.1592 & 4432.1 \\
\cline { 2 - 6 } & 2 & 20.2662 & 3445.6 & 20.5412 & 3592.5 \\
\cline { 2 - 6 } & 3 & 20.3322 & 3427.0 & 20.5412 & 3592.4 \\
\hline \multirow{4}{*}{4} & 1 & 14.1592 & 4430.9 & 14.1592 & 4431.7 \\
\cline { 2 - 6 } & 2 & 20.1152 & 3335.0 & 20.2944 & 3342.8 \\
\cline { 2 - 6 } & 3 & 20.1177 & 3314.5 & 20.2959 & 3403.3 \\
\hline
\end{tabular}

Table 1. Comparison between two accelerators.

In Table 2 a comparison between particle accelerators, based on a triangular cell array and an external hexagonal wall, is shown.

\begin{tabular}{|l|l|l|l|l|l|}
\hline & & \multicolumn{2}{|l|}{ Dielectric rods } & \multicolumn{2}{l|}{ Metallic rods } \\
\hline $\mathrm{N}$ & mode & $\begin{array}{l}\text { Frequency } \\
(\mathrm{GHz})\end{array}$ & $\mathrm{Q}$ & $\begin{array}{l}\text { Frequency } \\
(\mathrm{GHz})\end{array}$ & $\mathrm{Q}$ \\
\hline \multirow{3}{*}{2} & 1 & 14.9091 & 7018.3 & 14.1598 & 4439.0 \\
\cline { 2 - 6 } & 2 & 18.8251 & 6877.4 & 21.0130 & 4091.0 \\
\cline { 2 - 6 } & 3 & 19.1886 & 7128.9 & 21.0130 & 4091.7 \\
\hline \multirow{3}{*}{4} & 1 & 14.8314 & 7142.2 & 14.1592 & 4432.1 \\
\cline { 2 - 6 } & 2 & 18.4442 & 7020.8 & 20.5412 & 3592.5 \\
\cline { 2 - 6 } & 3 & 18.5926 & 7068.8 & 20.5412 & 3592.4 \\
\hline \multirow{4}{*}{4} & 1 & 14.8216 & 7163.0 & 14.1592 & 4431.7 \\
\cline { 2 - 6 } & 2 & 18.2596 & 7056.2 & 20.2944 & 3342.8 \\
\cline { 2 - 6 } & 3 & 18.3614 & 7096.1 & 20.2959 & 3403.3 \\
\hline
\end{tabular}

Table 2. Comparison between two accelerators, based on a triangular cell array and an external hexagonal wall. 
The two structures have been designed with dielectric and metallic rods, respectively. Of course, only two grating periods are required for localizing the first mode, thus reducing every further loss. The structure characterized by dielectric rods does not suffer from any reduction of performances due to the increase of the number of grating periods, both for the first mode and high order modes. In fact, the dielectric rods improve the quality factor with respect to the same structure with metallic rods, which are characterized by strong resistive losses.

Fig. 17 shows the $E_{z}$ field component distribution in the hexagonal cavity.

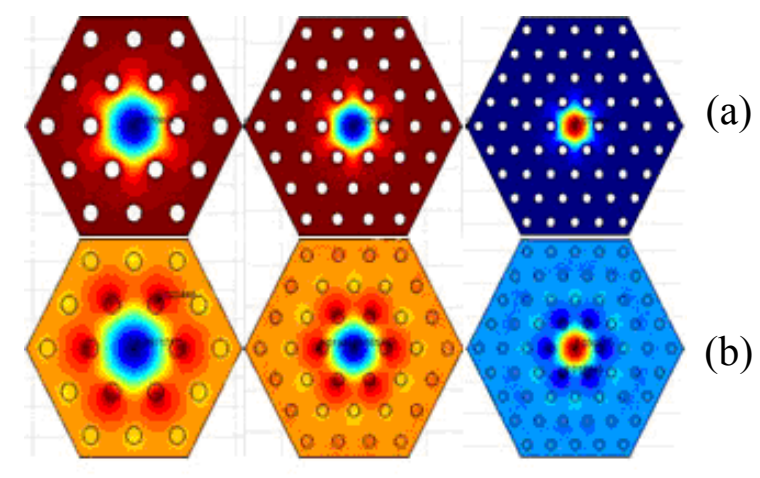

Fig. 17. First mode for metallic rods (a) and dielectric rods (b).

In Fig. 17 the first mode is shown in case of metallic rods (first row) for two, three and four grating periods. The same mode is sketched for dielectric rods (second row), thus showing a different field distribution. The same situation is depicted in Fig. 18, where the second order mode is shown. Because of field penetration inside columns, also losses due to dielectric medium have to be considered, according to Eqns. (9) and (10). However the losses due to the dielectric medium can be lower than the metallic ones, with improvement of the $Q$ factor, as demonstrated in Table 2.

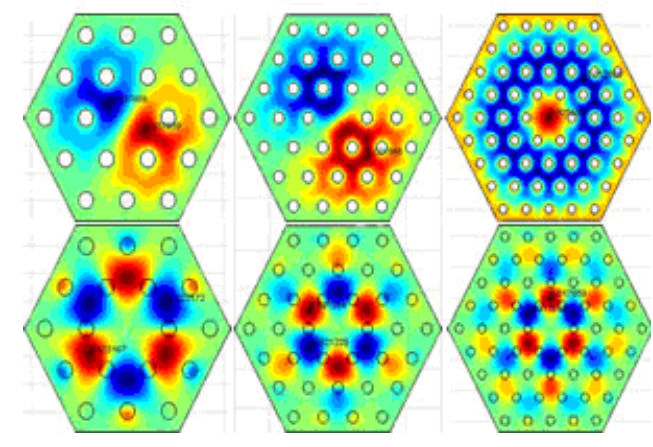

(a)

Fig. 18. Second mode for metallic rods (a) and dielectric rods (b). 


\section{Prototype realization and experimental measurements}

The copper prototype, shown in Fig. 19, has been realized by the Electronic Device Laboratory research group of Politecnico di Bari (Italy).

The difference between the theoretical results and those obtained by measures are related to the actual realization tolerances that, in this case, are limited to $0.1 \mathrm{~mm}$, and the inaccuracy of the experimental characterization. This implies that cylinders are placed in different position, not vertically aligned, with rough surfaces, etc.

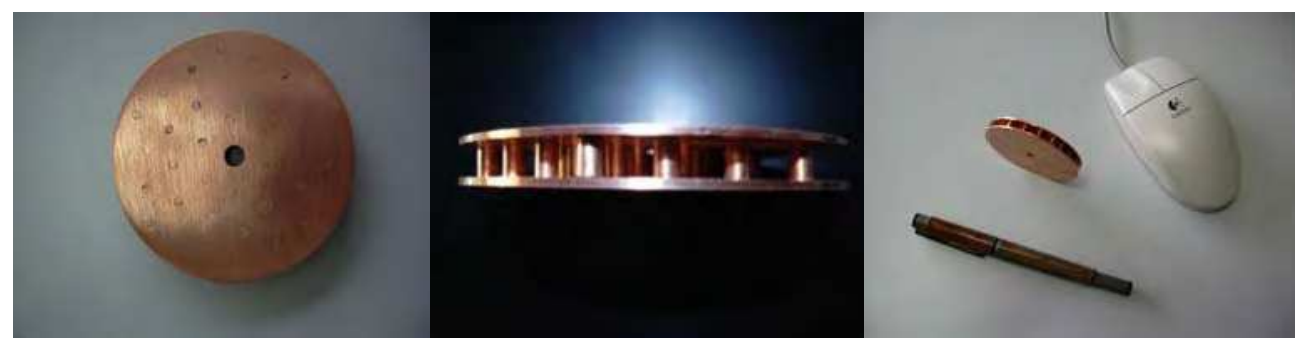

Fig.19. Prototype images. Dimensions are compared with a pen and a PC-mouse.

Secondly, the cavity is made of 36 cylinders enclosed between two copper plates. Thus the contact resistance between elements is added to the copper resistivity with an increasing value of losses with respect to the preliminary theoretical investigation and a consequent decrease of the $Q$-factor.

Finally, a $5 \mathrm{~mm}$ diameter hole has to be placed on each plate near the central defect region, in order to get the correct measures.

As shown in Fig. 20, the network analyzer HP 8720ES has been implemented to measure the s-parameters for the experimental characterization of the prototype.

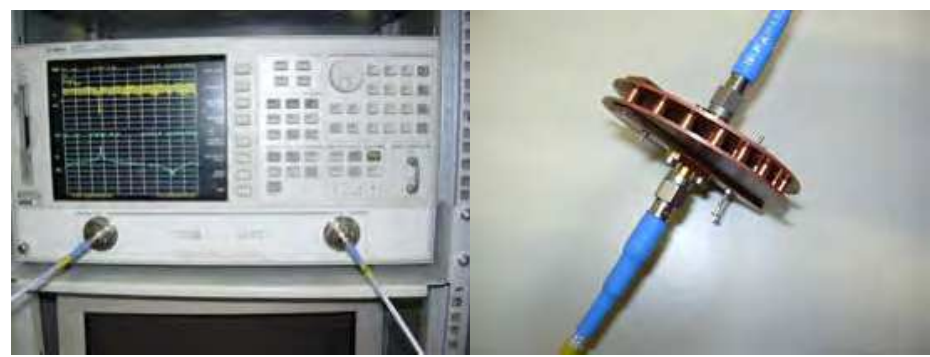

Fig. 20. Network Analyzer HP 8720ES with excitation and measure probes.

By setting the spectrum analyzer (Agilent Technologies, 2004) in a frequency range between 12 and $20 \mathrm{GHz}$ and a bandwidth at intermediate frequency (IF bandwidth) of $10 \mathrm{~Hz}$ (minimum value that allows to remove noise), the $s_{11}$ and $s_{21}$ parameters are measured, as shown in Fig.21. In this way the quality factor $Q$ of the first resonant mode (fundamental mode) is estimated as $\omega_{\text {ris }} / \Delta \omega_{-3 d B}$, where $\omega_{\text {ris }}$ is the angular frequency under resonant conditions and $\Delta \omega_{-3 d B}$ is the difference between the angular frequencies at the right and the 
left of the resonant frequency at which $s_{21}$ decreases of $3 \mathrm{~dB}$ with respect to the peak value. Thus the measured $Q$-factor is 352.98 .

The second resonant peak at $19.7 \mathrm{GHz}$, is smaller and wider than the first, placed at about 5 $\mathrm{GHz}$ of distance. The quality factor $Q$ of this peak is about 109.44 and, consequently, lower than that obtained for the first mode, as expected.
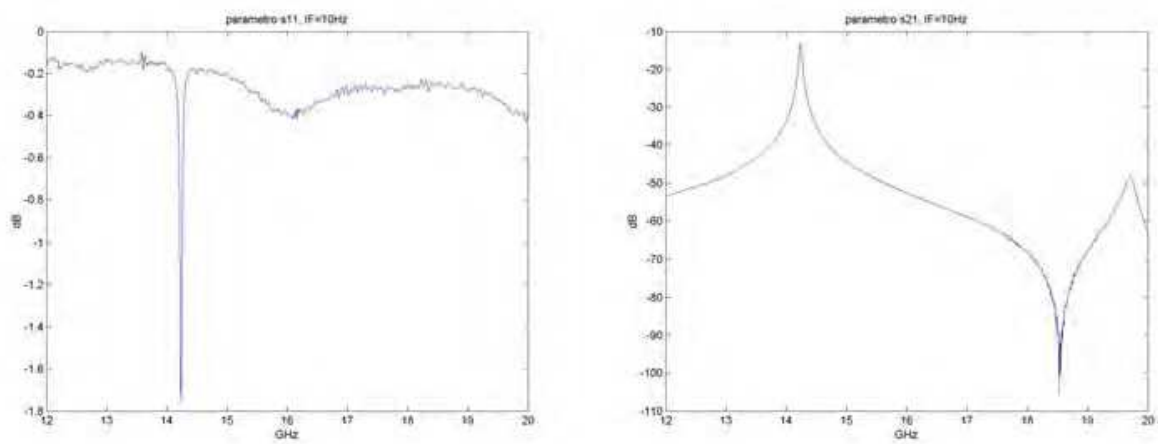

Fig. 21. Measured values of $s_{11}$ and $s_{21}$ between $12 \mathrm{GHz}$ and $20 \mathrm{GHz}$ with $\mathrm{IF}=10 \mathrm{~Hz}$.

\section{Conclusions}

We have investigated several structures in order to find the main geometrical parameters able to improve performances of a PBG based particle accelerator. All the simulations reveal good performances for a structure based on dielectric rods and a suitable number of grating periods.

A PBG-based resonant cavity has been designed, realized and measured for the first time in Europe. This cavity is able to accelerate hadrons in order to define the elementary unit cell of a high-efficiency and low-cost accelerator, whose sizes are smaller than the classical cyclotron, which is now used to accelerate hadrons with a lot of limitations.

The designed PBG accelerator will allow the attainment of important results in terms of therapy efficiency and feasibility, reaching a higher number of patients because of the reliability of the accelerator, which is the system kernel, and the falling implementation cost.

\section{References}

Agilent Technologies (2004). Exploring the architectures of Network Analyzers.

Coutrakon, G.; Slater, J. M.; Ghebremedhin, A. (1999). Design consideration for medical proton accelerators. Proceedings of the 1999 Particle Accelerator Conference, 1999, New York.

Dwoyer, D.L.; Hussaini, M.Y.; Voigt, R.G. (1988). Finite Elements - Theory and Application. Ed. Springer-Verlag, ISBN 0-387-96610-2, New York.

Kiriakidis, G. \& Katsarakis, N. (2000). Fabrication of 2D and 3D Photonic Bandgap Crystals in the $\mathrm{GHz}$ and THz regions. Mater. Phys. Mech., Vol. 1, pp. 20-26.

Perri, A. G. (2007). Introduzione ai dispositivi micro e nanoelettronici. Ed. Biblios, Vol. 1 - 2, ISBN 978-88-6225-000-9, Bari, Italy. 
Shapiro, M. A.; Brown, W. J.; Mastovsky, I.; Sirigiri, J. R.; Temkin, R. J. (2001). 17 GHz photonic band gap cavity with improved input coupling. Physical Review Special Topics-Accelerators and beams, Vol. 4, 2001, pp. 1-6.

Yablonovitch, E. (1994). Photonic crystals. Journal of Modern Optics, Vol. 41, n. 2, 1994, pp. 173-194. 


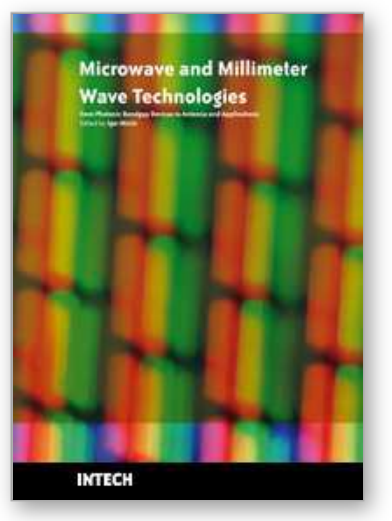

\author{
Microwave and Millimeter Wave Technologies from Photonic \\ Bandgap Devices to Antenna and Applications \\ Edited by Igor Minin
}

ISBN 978-953-7619-66-4

Hard cover, 468 pages

Publisher InTech

Published online 01, March, 2010

Published in print edition March, 2010

The book deals with modern developments in microwave and millimeter wave technologies, presenting a wide selection of different topics within this interesting area. From a description of the evolution of technological processes for the design of passive functions in milimetre-wave frequency range, to different applications and different materials evaluation, the book offers an extensive view of the current trends in the field. Hopefully the book will attract more interest in microwave and millimeter wave technologies and simulate new ideas on this fascinating subject.

\title{
How to reference
}

In order to correctly reference this scholarly work, feel free to copy and paste the following:

Roberto Marani and Anna Gina Perri (2010). Modelling and Design of Photonic Bandgap Devices: a Microwave Accelerating Cavity for Cancer Hadrontherapy, Microwave and Millimeter Wave Technologies from Photonic Bandgap Devices to Antenna and Applications, Igor Minin (Ed.), ISBN: 978-953-7619-66-4, InTech, Available from: http://www.intechopen.com/books/microwave-and-millimeter-wave-technologies-from-photonicbandgap-devices-to-antenna-and-applications/modelling-and-design-of-photonic-bandgap-devices-amicrowave-accelerating-cavity-for-cancer-hadronth

\section{INTECH}

open science | open minds

\section{InTech Europe}

University Campus STeP Ri

Slavka Krautzeka 83/A

51000 Rijeka, Croatia

Phone: +385 (51) 770447

Fax: +385 (51) 686166

www.intechopen.com

\section{InTech China}

Unit 405, Office Block, Hotel Equatorial Shanghai

No.65, Yan An Road (West), Shanghai, 200040, China 中国上海市延安西路65号上海国际贵都大饭店办公楼 405 单元

Phone: +86-21-62489820

Fax: +86-21-62489821 
(C) 2010 The Author(s). Licensee IntechOpen. This chapter is distributed under the terms of the Creative Commons Attribution-NonCommercialShareAlike-3.0 License, which permits use, distribution and reproduction for non-commercial purposes, provided the original is properly cited and derivative works building on this content are distributed under the same license. 